\title{
UTILIZAÇÃO DE MÉTODOS ESTATÍSTICOS MULTIVARIADOS NA CARACTERIZAÇÃO DO MOSAICO SUCESSIONAL EM FLORESTA SEMIDECÍDUAL ${ }^{1}$
}

\author{
Renata Cristina Batista Fonseca ${ }^{2}$, Ines Cristina de Batista Fonseca ${ }^{3}$
}

\begin{abstract}
RESUMO - O presente trabalho objetivou verificar a possibilidade da utilização de métodos estatísticos multivariados na caracterização das fases do desenvolvimento do mosaico sucessional de um trecho de floresta estacional semidecidual, através de variáveis estruturais. Foram alocadas parcelas de $10 \mathrm{~m}$ x $10 \mathrm{~m}$, em que se procedeu à análise estrutural, ou seja, levantamento fitossociológico acrescido das variáveis Porcentagem de Cobertura (PC), Altura do Dossel (AD) e Cobertura por Lianas (CL). Os métodos estatísticos empregados foram Análise de Componentes Principais e Análise de Agrupamento, mais especificamente Classificação Hierárquica Ascendente. O primeiro componente principal explicou 43,96\% da variância total, enquanto o segundo, 25,66\%. As variáveis Área Basal (AB), Diâmetro Médio (DM) e Dominância Média (DOM) apresentaram correlações positivas entre si superiores a 0,75 , podendo ser DM e DOM consideradas como um grupo de variáveis. As variáveis Número de Indivíduos (NI) e Número de Espécies (NE) apresentaram correlação 0,60, enquanto AD, CL e PC baixas correlações com as demais, indicando a importância da inclusão destas na análise. A classificação hierárquica e a partição dos grupos em quatro foram feitas considerando os dois primeiros eixos fatoriais. Os resultados indicaram dois comportamentos diferenciados: 1) valores baixos para AD e AB: Grupo 1, com valores baixos também para NI, NE e PC (fase de clareira); e Grupo 2, com valores elevados para NI e CL e baixos para DOM e DM (fase de construção); e 2) valores altos para AD e AB: Grupo 3, com valores altos também para NI, NE e PC e valor baixo para CL (fase madura); e Grupo 4, com valores elevados para DOM e DM e mais baixos para CL (fase de degradação). Os métodos estatísticos multivariados permitiram caracterizar as fases do desenvolvimento do mosaico sucessional, através das variáveis estruturais. A forma como foram estimadas as variáveis AD, CL e PC, porém, deve ser aprimorada, assim como é preciso incluir variáveis que discriminem melhor cada fase.
\end{abstract}

Palavras-chave: Métodos estatísticos multivariados, análise estrutural, mosaico sucessional, fitossociologia, floresta estacional semidecidual, análise de componentes principais, análise de agrupamento.

\section{MULTIVARIATE STATISTICAL METHODS FOR SUCCESSIONAL MOSAIC CHARACTERIZATION IN A SEMIDECIDUOUS FOREST}

\begin{abstract}
The aim of this paper was to verify the feasibility of using multivariate statistical methods through structural variables for successional mosaic characterization in a semideciduous forest section. Plots measuring $10 \mathrm{~m} \times 10 \mathrm{~m}$ were allocated for structural analysis (phytosociological survey plus the variables Coverage Percentage (CP), Canopy Height (CH) and Liana Cover (LC). The following statistical methods have been used: Principal Components Analysis and Cluster Analysis - more specifically, Ascending Hierarchical Classification. 43.96\%
\end{abstract}

\footnotetext{
${ }^{1}$ Recebido para publicação em 24.4.2002 e aceito para publicação em 08.6.2004

${ }^{2}$ Departamento de Recursos Naturais - Faculdade de Ciências Agronômicas - UNESP/Botucatu - Caixa Postal 237 - CEP: 18603-970. Botucatu-SP. E-mail: <rfonseca@fca.unesp.br>.

${ }^{3}$ Departamento de Agronomia - Centro de Ciências Agrárias - Universidade Estadual de Londrina - Caixa Postal 6001 CEP: 86051-990. Londrina-PR. E-mail: <inescbf@uel.br>.
} 
of the total variance has been explained by the first principal component and $25.66 \%$ by the second one. The variables Basal Area (BA), Average Diameter ( $A D)$ and Average Dominance (ADOM) presented positive correlations (higher than 0.75) among themselves. Therefore, AD and ADOM may be considered as variable groups. The variables Number of Individuals (NI) and Number of Species (NE) showed a 0.60 correlation. The variables $\mathrm{CH}, \mathrm{LC}$ and CP presented lower correlations. These findings show that their inclusion in this analysis was important. The hierarchical classification and the division of the groups in four parts have been performed considering two first factorial axes. Results showed two different types of behavior: 1) low values for CH and BA - Group 1 with low values also for NI, NE and CP (Gap Phase) and Group 2 with high values for NI and LC and low values for ADOM and AD (Building Phase); 2) high values for CH and BA - Group 3 with high values also for NI, NE and CP and low value for LC (Mature Phase) and Group 4 with high values for ADOM and AD, and lower for LC (Degradation Phase). The multivariate statistical methods allowed the forest mosaic developing phase characterization through structural variables. The estimative of $C H, L C$ and CP variables must be improved. Other variables should be included in order to better differentiate the phases.

Key words: Multivariate statistical methods, structural analysis, forest mosaic, phytosociology, semi-deciduous forest, principal components analysis, cluster analysis.

\section{INTRODUÇÃO}

Nos últimos anos, a compreensão da dinâmica de crescimento e desenvolvimento das florestas tropicais e dos processos de sucessão tem merecido maior atenção por parte dos estudiosos, principalmente em razão da destruição das florestas e da necessidade de definir parâmetros para o estabelecimento e manejo conservacionista de reservas e fragmentos florestais (ENGEL, 1993).

A floresta tropical possui como característica fundamental uma grande heterogeneidade ambiental no espaço e no tempo, de modo a constituir um mosaico formado por "retalhos” de diferentes idades, tamanhos e composição de espécies. Tal fato está relacionado à discussão sobre a sucessão secundária, já que esse mosaico conteria trechos em diferentes estágios sucessionais, sendo altamente importante para entender a diversidade e estrutura das florestas tropicais e, portanto, sua conservação (KAGEYAMA, 1987; WHITE e PICKETT, 1985).

O dossel de uma floresta muda à medida que as árvores crescem e morrem e outras as substituem. Esse equilíbrio dinâmico pode ser subdividido em três fases de crescimento/desenvolvimento florestal: a fase de clareira, a fase de construção e a fase madura. A fase de clareira, contendo mudas e árvores jovens, passa, à medida que cresce, pela fase de construção, que é uma floresta jovem e amadurece através de um crescimento contínuo de suas árvores constituintes (WHITMORE, 1978; 1990).

R. Árvore, Viçosa-MG, v.28, n.3, p. 351-359, 2004
Estudos de mosaicos sucessionais em florestas tropicais concentram-se, na maioria das vezes, em torno das aberturas naturais do dossel florestal causadas pela queda de árvores (dinâmica de clareiras) e nos processos de regeneração natural que se sucedem. Tais estudos estão baseados no conhecimento das espécies, o que é sempre um fator limitante em razão da alta riqueza de espécies e da diversidade de estratégias de ciclo de vida (ENGEL, 1993).

Diferentes espécies são beneficiadas em clareiras de diferentes tamanhos. O tamanho da clareira, dessa forma, tem uma importante influência na composição de espécies e no arranjo espacial da floresta (WHITMORE, 1978).

Outra forma de estudar os ciclos de desenvolvimento e regeneração do mosaico sucessional é através da silvigênese. Este termo se refere ao processo pelo qual a arquitetura da floresta é construída, ou seja, ao processo de “fabricação da floresta” (HALLÈ et al., 1978).

A seqüência de desenvolvimento de uma ecounidade, ou seja, unidade de regeneração coetânea e ecologicamente uniforme, passa por uma fase de reorganização, uma fase de desenvolvimento, uma fase bioestática ou em equilíbrio dinâmico e uma fase de degradação. O “evento zero”, que marca o aparecimento de uma ecounidade nova na floresta, é a formação de uma clareira. Assim, o desenvolvimento das ecounidades é tratado como um subprocesso do desenvolvimento da unidade sil- 
vática ou mosaico florestal (OLDEMAN, 1989,1990).

O tamanho original da ecounidade irá determinar seu comportamento subseqüente, o tipo de processo dominante e as estratégias de ciclo de vida das espécies que a compõem. Assim, ecounidades grandes passam por um processo completo de sucessão secundária (TORQUEBIAU, 1986), desde a fase de clareira, passando pelas fases de construção e de maturidade (WHITMORE, 1978), enquanto ecounidades pequenas são ocupadas principalmente por árvores suprimidas.

A caracterização das ecounidades, baseada na arquitetura arbórea, permite não só estudar a evolução temporal (dinâmica sucessional) de eventos passados que determinam o estado atual, mas também fazer previsões acerca do potencial futuro de regeneração e auto-sustentabilidade do ecossistema (ENGEL, 1993).

Outro método para a caracterização das fases de desenvolvimento do mosaico sucessional, utilizado em fragmentos florestais, proposto por Tabanez et al. (1997), é através do Índice de Desenvolvimento Sucessional - IDS. Este índice leva em conta os seguintes parâmetros: altura das maiores árvores, índice de diversidade de Shannnon-Weaver, soma do VC (Valor de Cobertura) das espécies não-pioneiras e área basal das maiores árvores. Esses parâmetros são igualados ao seu valor máximo (valor relativo de 100) dentro do fragmento, para que todos tenham o mesmo valor relativo e possam ser somados para formar o parâmetro composto (IDS = altura + ID + IVC + área basal), que pode somar o valor máximo de 400. O IDS será menor quanto menos madura for a floresta ou maior quanto mais avançado for o estágio da sucessão (TABANEZ et al., 1997).

Levantamentos fitossociológicos em florestas tropicais vêm sendo muito utilizados para retratar a estrutura de determinados trechos de mata, mas são raros os que retratam as variações que ocorrem nas diferentes fases de desenvolvimento do mosaico florestal (GANDOLFI et al., 1995).

O presente trabalho teve como objetivo verificar a possibilidade da utilização de métodos estatísticos multivariados na caracterização das fases de desenvolvimento do mosaico sucessional de um trecho de floresta estacional semidecidual, através de variáveis estruturais.

\section{MATERIAL E MÉTODOS}

\subsection{Análise Estrutural}

O trabalho foi realizado no interior de um fragmento de floresta secundária tardia alta, de aproximadamente 60 ha, denominado "Mata da Bica”, localizado na Fazenda Experimental Edgardia, pertencente à Universidade Estadual Paulista - UNESP/Campus de Botucatu (latitude de $22^{\circ} 49^{\prime} \mathrm{S}$, longitude de $48^{\circ} 23^{\prime} \mathrm{W}$ e altitude de $577 \mathrm{~m}$ ), situada no município de Botucatu, SP.

No Estado de São Paulo, nos levantamentos fitossociológicos tem-se adotado como critério de inclusão um diâmetro mínimo de $5 \mathrm{~cm}$ na altura do peito (DAP), para formações florestais com fisionomia característica de mata (RODRIGUES, 1988). De forma a se ter um número suficiente de indivíduos por parcela, o tamanho de parcela adotado em geral é de $100 \mathrm{~m}^{2}$ $(10 \times 10 \mathrm{~m})$ ou $200 \mathrm{~m}^{2}$ (10 x $\left.20 \mathrm{~m}\right)$. As parcelas retangulares são mais eficientes que as quadradas e as circulares devido à tendência de agrupamento na vegetação. Porém, para análise do mosaico florestal, quanto menor for a parcela, maior será a chance de se ter uma unidade homogênea em relação às fases de desenvolvimento do mosaico sucessional.

Dessa forma, foram instaladas 200 parcelas de 10 x 10 m no centro do fragmento, formando uma malha de 100 x 200 m, onde se procedeu à análise estrutural (levantamento fitossociológico acrescido do estudo das variáveis Porcentagem de Cobertura, Altura do Dossel e Cobertura por Lianas), em 100 parcelas sorteadas.

A metodologia empregada no levantamento fitossociológico, bem como no estudo das variáveis anteriormente citadas, pode ser encontrada em Fonseca (1998) e Fonseca e Rodrigues (2000).

\subsection{Análise de Componentes Principais e Classificação Hierárquica Ascendente}

A análise dos componentes principais dos dados de medidas estruturais das parcelas, as quais constituem as unidades amostrais, e a classificação hierárquica foram feitas através do programa SPAD, versão 3.5. (CISIA-CERESTA, 1998).

Essas técnicas exploratórias foram utilizadas com o objetivo de agrupar as parcelas semelhantes quan-

R. Árvore, Viçosa-MG, v.28, n.3, p. 351-359, 2004 
to às características estruturais e não para testar hipóteses. A corroboração da hipótese de que os grupos formados consistem nas fases do desenvolvimento sucessional foi feita através da contraposição com os resultados de Fonseca e Rodrigues (2000), que serviram como referencial teórico dos grupos.

\subsubsection{Análise de Componentes Principais}

Considerem-se os dados uma amostra de $\mathrm{n}$ unidades em cada uma das $p$ variáveis, $X_{j}(j=1, \ldots, p)$. Os dados podem ser escritos na forma matricial como:

$$
\mathrm{X}=\left[\mathrm{X}_{\mathrm{ij}}\right],
$$

em que $X_{i j}$ corresponde ao valor na j-ésima variável para a i-ésima unidade amostral $(i=1, \ldots, n)$.

O objetivo do ACP é transformar a matriz X de $p$ variáveis, que podem estar correlacionadas em uma matriz Y de p variáveis hipotéticas não-correlacionadas, que decrescem em variância da primeira para a última (MAXWELL, 1977).

A primeira coluna de $\mathrm{Y}$ representa o primeiro componente principal (variável $\mathrm{y}_{1}$ ). É uma soma ponderada das variáveis $X_{j}(j=1, \ldots, p)$ e pode ser escrita como:

$$
\mathrm{y}_{1}=\mathrm{X}_{1} \mathrm{u}_{11}+\mathrm{X}_{2} \mathrm{u}_{21}+\ldots+\mathrm{X}_{\mathrm{p}} \mathrm{u}_{\mathrm{p} 1}
$$

em que cada $\mathrm{u}_{\mathrm{j} 1}$ é o peso da j-ésima variável nesse componente. Para a amostra de $\mathrm{n}$ unidades, a notação matricial é:

$$
\mathrm{y}=\mathrm{Xu}
$$

em que y é o vetor nx 1 dos valores em $y_{i}(i=1, \ldots$, n) e u é o vetor px1 dos pesos que devem ser determinados.

A variância de $\mathrm{y}_{1}$ é a raiz característica $\lambda_{1}$. A soma de todas as raízes corresponde à variância total; então, o componente principal $\mathrm{y}_{1}$ explica, em porcentagem, a porção da variação:

$$
\left(\lambda_{1} / \sum \lambda_{i}\right) \times 100 \quad(i=1, \ldots, n)
$$

Quando as variáveis observadas são inter-relacionadas, em geral os cinco primeiros componentes explicam grande parte da variância sem séria perda de informação, e as variáveis observadas podem ser substituídas por um pequeno conjunto de variáveis, o que é muito importante como procedimento prévio de outras análises, por exemplo no caso de técnicas de análise de agrupamento apropriadas quando o número de variáveis é pequeno (MAXWELL, 1977).

Quando as variáveis estudadas $X_{j}(j=1, \ldots, p)$ têm a mesma unidade de medida e dimensões não muito discrepantes, o método indicado é razoável e aconselhável. Mas, às vezes, as unidades são inteiramente diferentes. Em tais casos, é recomendado usar as variáveis reduzidas, isto é, cada uma dividida pelo respectivo desvio-padrão. Isso é equivalente a considerar a matriz de correlações.

O tipo de ACP utilizado foi o que consiste na decomposição fatorial da variância da matriz de correlações entre as variáveis ativas, para neutralizar o efeito das unidades de medida.

\subsubsection{Classificação Hierárquica Ascendente}

A técnica de agrupamento utilizada foi a classificação hierárquica ascendente. O método hierárquico aglomerativo ou ascendente começa com os objetos individuais, denominados parcelas neste trabalho. Então, têm-se, inicialmente, tantos grupos quantos forem os objetos. Os mais semelhantes são agrupados, sendo estes grupos iniciais fundidos de acordo com suas similaridades. Eventualmente, como as semelhanças diminuem, todos os subgrupos são fundidos em um único. Os resultados podem ser mostrados na forma de diagrama bidimensional, conhecido como dendrograma.

O algoritmo (processo interativo) de agregação utilizado foi o método baseado no crescimento mínimo do momento de ordem 2. O método, em lugar de reunir as duas classes que apresentam a menor distância (semelhança), agrega duas classes, de tal maneira que a classe resultante tenha dispersão mínima com relação a todas as classes que possam ser formadas em uma etapa do seu algoritmo. Em vez de calcular a distância entre as classes, o algoritmo calcula a dispersão de cada nova classe eventualmente constituída de duas classes originais. Para aplicar esse método, é necessário que a distância entre os objetos a classificar seja uma quadrática $\mathrm{d}^{2}$ (euclidiana, euclidiana reduzida ou distância do $\mathrm{c}^{2}$ ).

No caso de uma tabela $T_{(n, p)}$ de variáveis quantitativas, a estratégia de agregação do crescimento mínimo do momento de ordem 2 é chamado de método de Ward. Se são agrupadas as classes $\{k\}$ e $\left\{k^{\prime}\right\}$, o aumento da variância intragrupo, também chamado de nível de agregação $\mathrm{D}_{(\mathrm{k}} \cup_{\mathrm{k}^{\prime}}$ ) é definido por 


$$
\Delta_{\left(\mathrm{k} \cup \mathrm{k}^{\prime}\right)}=\frac{\mathrm{n}_{\mathrm{k}} \cdot \mathrm{n}_{\mathrm{k}^{\prime}}}{\mathrm{n}_{\mathrm{k}}+\mathrm{n}_{\mathrm{k}^{\prime}}} \mathrm{d}_{\left(\mathrm{G}_{\mathrm{k}}, \mathrm{G}_{\mathrm{k}^{\prime}}\right)}^{2}
$$

em que $G_{k}$ e $G_{k}$, correspondem aos centros de gravidade das classes k e k' (CRIVISQUI, 1999b).

A partir de um dendrograma, pode-se escolher uma partição dos n objetos submetidos à classificação hierárquica ascendente. Para selecionar uma boa partição, deve-se escolher um nível de agregação para o qual o valor não seja muito elevado, ou seja, baixa transformação das distâncias iniciais entre os objetos. Para isso, é suficiente "cortar" o dendrograma com uma reta que cruze os ramos ascendentes mais compridos.

\section{RESULTADOS}

As análises permitiram avaliar a relação entre as características estruturais e a semelhança entre as parcelas, além do estabelecimento dos grupos de parcelas homogêneas.

O primeiro componente principal, combinação linear das oito variáveis avaliadas, explicou 43,96\% da variância total. O segundo componente principal explicou 25,66\% da variância total, representando, os dois primeiros eixos fatoriais, $69,62 \%$ da variância total. O terceiro componente principal esclareceu $11,39 \%$ dos suplementares da variância total, correspondendo a um eixo de baixo grau de generalidade.

As parcelas são representadas por pontos no espaço formado pelos eixos fatoriais. A proximidade entre dois pontos significa a semelhança dos valores das variáveis dessas parcelas. As variáveis são representadas por vetores que podem ser representados no espaço de representação dos indivíduos (Figura 1).

A qualidade de representação de uma variável é dada pela proximidade do extremo desta ao círculo de raio 1, o que pode ser visto na Figura 2. A correlação entre duas variáveis é representada pelo ângulo que os vetores correspondentes formam.

As variáveis Área Basal, Diâmetro Médio e Dominância Média apresentaram correlações positivas entre si superiores a 0,75 , podendo ser Diâmetro Médio e Dominância Média considerados um grupo de variáveis. As variáveis Número de Indivíduos e Número de Espécies apresentaram correlação de 0,60.

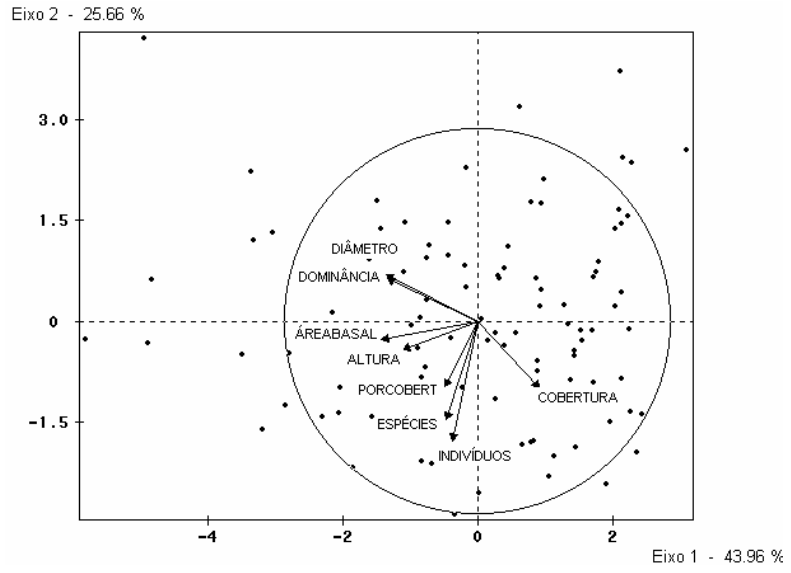

Figura 1 - Representação das parcelas e das variáveis no primeiro plano fatorial (Representation of plots and variables in the first pair of principal components).

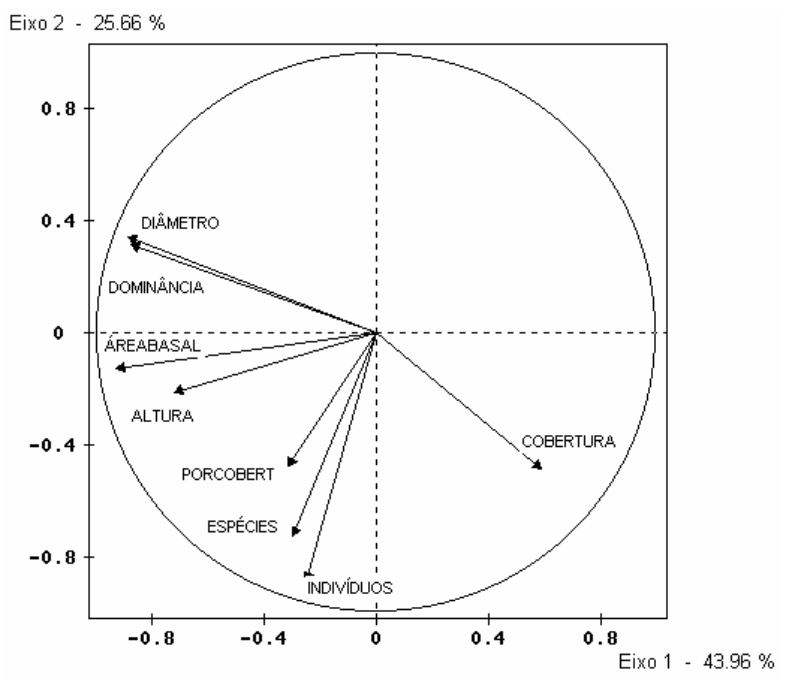

Figura 2 - Representação da qualidade de representação das variáveis (Representation of the variable representation qualities).

As baixas correlações entre a variável Altura e as demais, entre a variável Cobertura por Lianas e as demais e entre a variável Porcentagem de Cobertura e as demais evidenciaram que foi importante a inclusão dessas correlações na análise.

A classificação hierárquica e a partição dos grupos em quatro foram feitas considerando os dois primeiros

R. Árvore, Viçosa-MG, v.28, n.3, p. 351-359, 2004 
eixos fatoriais. A partição em quatro grupos foi definida através do dendrograma (Figura 3) e do valor do índice referente ao nível de agregação.

De modo geral, os resultados indicaram dois comportamentos diferenciados (Figura 4):

1) Grupos 1 e 2 - Valores baixos para Altura e Área Basal: o Grupo 1, com valores baixos também para Número de Indivíduos, Número de Espécies e Porcentagem de Cobertura; e o Grupo 2, com valores elevados para Número de Indivíduos e Cobertura por Lianas e baixos para Dominância Média e Diâmetro Médio.

2) Grupos 3 e 4 - Valores altos para Altura e Área Basal: o Grupo 3, com valores altos também para Número de Indivíduos, Número de Espécies e Porcentagem de Cobertura e valor baixo para Cobertura por Lianas; e o Grupo 4, com valores elevados para Dominância Média e Diâmetro Médio e mais baixos para Cobertura por Lianas.

Na Tabela 1 apresentam-se as médias e desviospadrão das variáveis estudadas da área total e de cada grupo de parcelas. O programa utilizado para a análise dos dados exibe somente essas medidas descritivas das variáveis que realmente caracterizam cada grupo.

De acordo com as características apresentadas, os grupos podem ser associados às seguintes fases de desenvolvimento do mosaico sucessional:

1) Fase de clareira - Grupo 1.

2) Fase de construção - Grupo 2.

3) Fase madura - Grupo 3.

4) Fase de degradação ou supermadura-Grupo 4.

Utilizando o método de agrupamento multivariado K-médias para mesmo conjunto de dados, Fonseca e Rodrigues (2000) encontraram resultados semelhantes.

Uma diferença nos resultados dos agrupamentos ocorreu na classificação de parcelas que apresentavam altos valores de Diâmetro Médio e Dominância Média e valor médio a alto de Área Basal, que são características de fase madura; e valores baixos para Número de Indivíduos, Número de Espécies, Porcentagem de Cobertura e Cobertura por Lianas, que são características da fase de clareira. No método utilizado neste

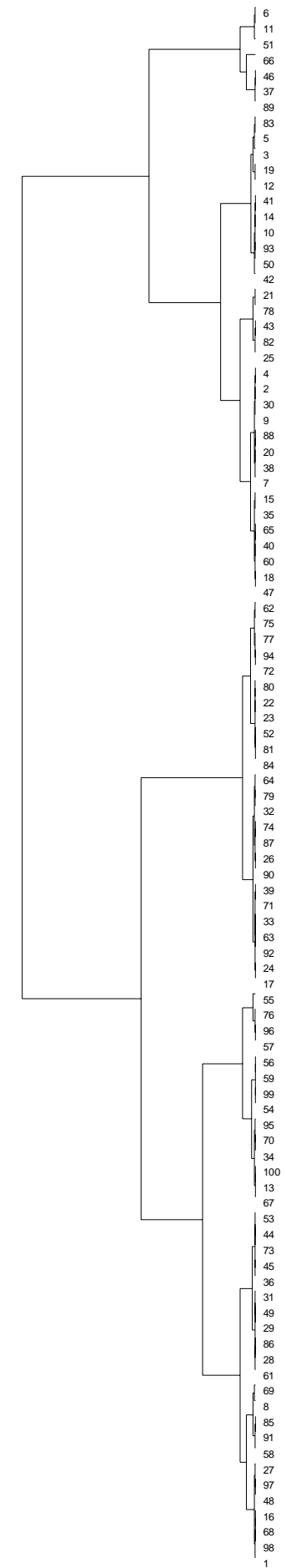

Figura 3 - Classificação hierárquica ascendente sobre os dois primeiros eixos fatoriais (dendrograma) (Ascending hierarchical classification on the two first factorial axes (dendrograms)). 


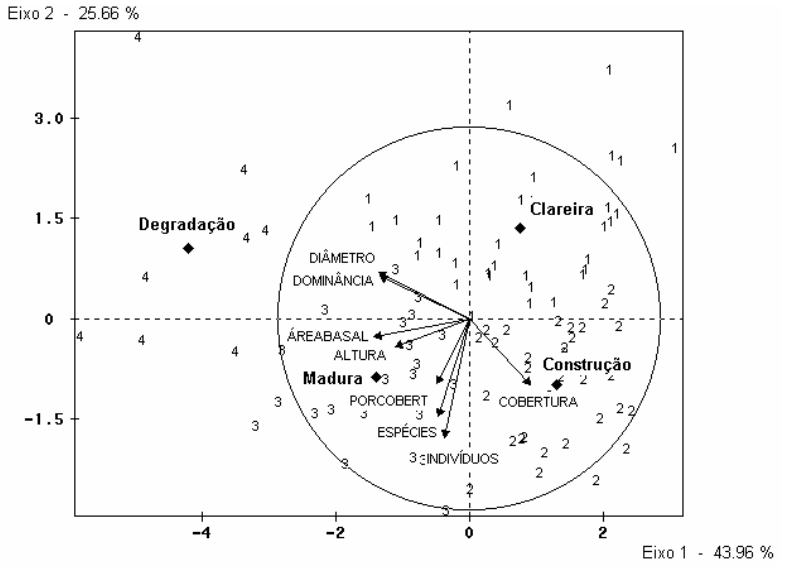

Figura 4 - Representação das parcelas classificadas e das variáveis no primeiro plano fatorial (Representation of the classified plots and variables in the first factorial plan). algumas das variáveis foram obtidas. A Altura do Dossel foi estimada considerando-se somente os cinco maiores indivíduos; assim, parcelas em fase de clareira, mas com os poucos indivíduos altos, apresentam valor alto de altura, podendo ser confundida numericamente com a fase madura. A Porcentagem de Cobertura foi tomada em 13 pontos da parcela, sendo oito nas bordas, o que pode estar representando a situação de parcelas vizinhas; outro aspecto é que essa cobertura não é somente do dossel de espécies arbóreas, mas também de lianas. Assim, parcelas em fase de clareira, mas com grande presença de lianas, apresentaram altos valores de Porcentagem de Cobertura, podendo ser confundida numericamente com fase madura.

Outra diferença nos resultados dos agrupamentos foi na classificação de parcelas que apresentavam baixos valores de Diâmetro Médio e Dominância Média e valor alto de Cobertura de Lianas, que são

Tabela 1 - Médias e desvios-padrão das variáveis estudadas da área total e de cada grupo de parcelas (Means and standard deviations for the studied variables of total area, and for each group of plots).

\begin{tabular}{|c|c|c|c|c|c|}
\hline & Área Total & Grupo 1 & Grupo 2 & Grupo 3 & Grupo 4 \\
\hline Altura (m) & $10,04 \pm 2,45$ & $9,15 \pm 2,24$ & $8,80 \pm 1,64$ & $12,17 \pm 1,81$ & $12,11 \pm 1,95$ \\
\hline Área basal $\left(\mathrm{m}^{2}\right)$ & $0,27 \pm 0,21$ & $0,17 \pm 0,09$ & $0,15 \pm 0,07$ & $0,41 \pm 0,13$ & $0,75 \pm 0,23$ \\
\hline $\begin{array}{l}\text { Número de } \\
\text { Indivíduos }\end{array}$ & $11,04 \pm 3,76$ & $7,50 \pm 2,02$ & $12,42 \pm 2,72$ & $14,24 \pm 2,85$ & \\
\hline $\begin{array}{l}\text { Cobertura } \\
\text { por Lianas }\end{array}$ & $17,94 \pm 10,37$ & & $28,22 \pm 9,03$ & $12,52 \pm 6,39$ & $6,12 \pm 3,10$ \\
\hline $\begin{array}{l}\text { Número de } \\
\text { Espécies }\end{array}$ & $6,09 \pm 1,60$ & $4,82 \pm 1,12$ & & $7,08 \pm 1,52$ & \\
\hline $\begin{array}{l}\text { Diâmetro } \\
\text { Médio (cm) }\end{array}$ & $13,73 \pm 3,87$ & & $10,69 \pm 1,29$ & & $22,58 \pm 3,87$ \\
\hline $\begin{array}{l}\text { Porcentagem } \\
\text { de Cobertura }\end{array}$ & $97,37 \pm 2,27$ & $95,92 \pm 3,27$ & & $98,44 \pm 0,59$ & \\
\hline $\begin{array}{l}\text { Dominância } \\
\text { Média }\end{array}$ & $0,03 \pm 0,02$ & & $0,01 \pm 0,00$ & & $0,08 \pm 0,02$ \\
\hline $\begin{array}{l}\text { Número de } \\
\text { Parcelas }\end{array}$ & 100 & 34 & 33 & 25 & 8 \\
\hline
\end{tabular}

trabalho, essas parcelas foram classificadas como clareira, enquanto no método K-médias, maduras. Acredita-se que o problema não esteja ligado ao método de agrupamento e sim à ausência de variáveis que discriminem tais parcelas, associadas à maneira como características de fase de construção, mas com valores intermediários de Porcentagem de Cobertura, Número de Espécies e Número de Indivíduos, que são características de transição entre clareira e construção. No método utilizado neste trabalho, tais parce- 
las foram classificadas como construção, enquanto no método K-médias, como clareira. Nesse caso, acreditase que o problema seja a ausência de variáveis que discriminem essas parcelas nas duas fases ou em uma nova fase transicional. Nota-se porém, de acordo com o dendrograma, que, se fosse realizada uma partição em cinco grupos, ter-se-ia a subdivisão do Grupo 1, o que caracterizaria uma fase de superclareira e não a subdivisão da fase de construção, reforçando a necessidade de inclusão de novas variáveis.

\section{CONCLUSÕES}

A utilização, de forma combinada, dos métodos estatísticos multivariados Análise de Componentes Principais e Classificação Hierárquica Ascendente permitiu caracterizar as fases do desenvolvimento do mosaico sucessional, através das variáveis estruturais, porém são necessárias as seguintes considerações:

* A forma como foram estimadas as variáveis Altura, Cobertura por Lianas e Porcentagem de Cobertura deve ser aprimorada.

* A busca de variáveis que discriminem melhor as fases de desenvolvimento do mosaico sucessional deve ser incrementada.

* O próprio caráter gradativo de desenvolvimento do mosaico sucessional dificulta a classificação em poucas fases. Assim, embora o método seja aprimorado, sempre existirão situações intermediárias caraterísticas de transição.

\section{REFERÊNCIAS BIBLIOGRÁFICAS}

CISIA - CERESTA. Programa: SPAD for Windows (software). Versão 3.5, 1998. (CD-ROM).

\section{CRIVISQUI, E. Apresentação da análise em componentes principais. Londrina: UEL, 1999a. 31 p. (Apostila)}

CRIVISQUi, E. Apresentação do métodos de classificação. Londrina: UEL, 1999b. 57 p. (Apostila)

ENGEL, V.L. Silvigênese, dinâmica de fragmentos florestais e a conservação de florestas tropicais. Série Técnica Florestal, v. 1, n. 1, p. 21, 1993.

R. Árvore, Viçosa-MG, v.28, n.3, p. 351-359, 2004
FONSECA, R.C.B. Fenologia e estrutura de uma floresta semidecídua, em Botucatu-SP: relação com as fases de desenvolvimento sucessional. 1998. $198 \mathrm{f}$. Dissertação (Mestrado em Ciências Florestais) Escola Superior de Agricultura Luiz de Queiroz, Piracicaba, 1988.

FONSECA, R.C.B.; RODRIGUES, R.R. Análise estrutural e aspectos do mosaico sucessional de uma floresta semidecídua em Botucatu,SP.

Scientia Forestalis, n. 57, p. 27-43, 2000.

GANDOLFI, S.; LEITÃO FILHO, H.; BEZERRA, C.L.F. Levantamento florístico e caráter sucessional das espécies arbustivo-arbóreas de uma floresta mesófila semidecídua no município de Guarulhos-SP. Revista Brasileira de Botânica, v. 55, n. 4, p. 753-767, 1995.

HALLÉ, F.; OLDEMAN, R.A.A.; TOMLINSON, P.B. Tropical treesand forests. Berlin: Springer-Verlag, 1978. $441 \mathrm{p}$.

KAGEYAMA, P.Y. Conservação "in situ” de recursos genéticos de plantas. IPEF, v. 35, p. 7 37, 1987.

MAXWELL, A. E. Principal component analysis. In:--_--. Multivariate analysis in behavioural research. London: Chapman and Hall, 1977. p. 39-45.

OLDEMAN, R.A.A. Dynamics in tropical rainforests. In: HOLM-NIELSEN, L.B.; NIELSEN, I.C.; BALSLAV, H. Tropical forest: botanical dynamics, speciation and diversity. London: Academic Press, 1989. p. 3-21.

OLDEMAN, R.A.A. Elements of silvology. New York: Spriger-Verlag, 1990. 623 p.

RODRIGUES, R.R. Métodos fitossociológicos mais usados. Casa da Agricultura, v. 10, n. 1, 1988. $5 \mathrm{p}$.

TABANEZ, A.A.J.; VIANA, V.M.; DIAS, A.S. Conseqüências da fragmentação e do efeito de borda sobre a estrutura, diversidade e sustentabilidade de um fragmento de floresta de planalto de Piracicaba, SP. Revista Brasileira de Biologia, v. 57, n. 1, p. 47-60, 1997. 
TORQUEBIAU, E.F. Mosaic patterns in dipterocarp rain forest in Indonesia and their implications for pratical forestry. Tropical Ecology, v. 2, p. 301-325, 1986.

WHITE, P.S.; PICKETT, S.T.A. Natural disturbance and patch dynamics: an introduction. In: WHITE, P.S.; PICKETT, S.T.A. (Eds.). The ecology of natural disturbance and patch dynamics. New York: Academic Press, 1985. p. 3-13.
WHITMORE, T.C. An introduction to tropical rain forest. New York: Oxford University Press, 1990. 226 p.

WHITMORE, T.C. Gaps in the forest canopy. In: TOMLINSON, P.B.; ZIMMERMANN, M.H. Tropical trees as living system. Cambrigde: Cambrige University Press, 1978. p. 639-655. 